\title{
The Potential Contamination Scenarios simulation in Case of the Water Chlorination Technological Process Violation in the Urban Environment
}

\author{
Irina Lapshina ${ }^{1,{ }^{*}}$, Olga Usenko ${ }^{2}$, and Alena Zhidkova ${ }^{1}$ \\ ${ }^{1}$ Rostov State University of Economics, Bolshaya Sadovaya st., 69, Rostov-on-Don 344002, Russia \\ ${ }^{2}$ Institute of Radio Engineering Systems and Control Southern Federal University, 105/42, \\ Bolshaya Sadovaya Str., Rostov-on-Don, 344006, Russia
}

\begin{abstract}
The research raise the question of the need to analyse and model the processes associated with the emission of the emergency-chemical hazardous substance (ECHS) in the urban environment. Because the assessment of the environmental pollution, the early and prompt prediction of the scale of contamination in the event of releases is the potent poisonous substance (PPS) into the environment in case of accidents (destructions) at chemically hazardous facilities remains an urgent issue. We emphasize that in order to ensure the life of any settlement, there is a need for the availability of drinking water that meets sanitary standards and hygienic rules. Chlorination is traditionally used in most of the country's settlements. To take timely measures to eliminate accidents with the emission of chlorine, the most accurate results of calculating the main indicators are necessary, such as the depth of the zone of contamination of PPS, the amount of the poisonous substance in primary and secondary clouds, the area of contamination, etc. The method of calculating these indicators is based on the use of reference tables and the coefficient method, which are convenient enough for the manual method. However, in emergencies, when serious threats to the life and health of people arise, and the situation can deteriorate rapidly, there is an acute shortage of time for a general assessment of the situation, making decisions on the organization of the rescue measures. It is obvious that the staff simply will not have enough time for scrupulous calculations, and a stressful situation, in addition, with a high degree of probability will provoke serious errors and inaccuracies.
\end{abstract}

\section{Introduction}

At the beginning of our study, we note that the potent poisonous substance (PPT) - chlorine is stored in containers, as a rule of a large volume (for example, 2 containers of 0.9 tons each). Moreover, taking into account the fact that chlorine is a potent poisonous substance

\footnotetext{
*Corresponding author: ira_lapshina_2015@mail.ru
} 
[1] and has a highly directed mechanism of action, these requirements lead to the need for an increased level of attention to the conditions of its storage and measures to prevent accidents, as well as to conduct rescue and urgent work in the emergency zone. The size of the center of the chemical damage depends on the scale and method of their application. For example, it is an emergency depressurization of containers with the emergencychemical hazardous substance (ECHS), meteorological conditions, as well as the terrain and its development. At the time of application, toxic substances pass from a liquid or solid state into a droplet-liquid, gaseous, vaporous or aerosol (fog, smoke).

Practice shows that the main causes of the emergency are:

1. the possibility of a fire in the transformer room based on the real situation at a potentially hazardous facility (water treatment plant) and structural elements of the building;

2. the depressurization of valves, flanged and welded joints;

3. The mechanical damage of the tank and pipeline equipment, the corrosive and thermal effects on it;

4. the ingress of the extraneous substances (hydrogen, hydrocarbons, water, etc.) into the vessels with the liquid chlorine;

5. the defects and fatigue phenomena in metal and welded elements of vessels and pipelines;

6. the errors made in the design, manufacture, installation, repair and performance of the technological operations in the process of production, storage and consumption of chlorine.

Note that back in the Soviet Union and earlier in the pre-revolutionary period in the history of the Russian state, much attention was paid to combating fires and especially to their prevention. Many analysts analyzed these questions at the theoretical and practical levels: M. A. Balabanov, K. I. Berzin, V. N. Vinogradov, S. G. Golubev, M. A. Ermolova, G. I. Zhukov, E. K Krivosheeva, A. V. Lukovnikov, A. A. Lugovoi, A. A. Rubin, N. P. Trebezov, N. D. Shebeko, I. M. Shcherbakov, N. N. Shablov and others.

The authors investigate the issues of carrying out the emergency rescue and other urgent work in the emergency zone: I.V. Andrianova, S.V. Vlasov, A.V. Epikhin, M.I. Kamyshansky, A.D. Logoshin, L.G. Odintsov, V. Ya. Perevoshchikova, M. I. Falyuta, S. K. Shoigu, V. I. Yurtushkin and others.

In addition, the attention was paid to the issues related to the extinguishing fires in the urban and rural areas on the pages of pre-revolutionary magazines. Therefore, for example, this is the Russian periodical "Fireman", and then the Soviet edition "Fire business". Currently, the list of journals and the issues studied on their pages has expanded: the scientific and technical journal "Fire Safety", the network scientific journal "Topical Issues of Fire Safety", the journal catalog "Fire Regime", the All-Russian specialized journal "Safety" and some other periodic editions.

Note that the emergency associated with the fire by the civilians and professional rescuers is interpreted differently. So, the emergency situation for the personnel of the fire departments is the situation in which there is or is already developing a threat to the life and health of the participants in extinguishing the fire [2].

The development of the fire and the nature of its impact on the various objects is a complex physical process. Heat transfer from the combustion source to the enclosing structures mainly occurs due to the convective flows of the combustion products, radiation, thermal conductivity and the direct effect of the flame on the structures. The mechanism of the thermal interaction between the hearth and structures depends on the relative position and geometric dimensions of the torch and the structures themselves. The characteristics of the combustible load, the properties of the combustion products, the thermal characteristics of structures, as well as the nature of gas exchange affect the magnitude of the thermal interaction of the hearth with the structures [3]. 


\section{Main part}

It is known that the basis of the action of the emergency-chemical hazardous substances that manifest their properties when directly exposed to people and animals, finding them in an infected atmosphere and in contact with an infected area, equipment and other objects located in this area. Poisonous substances also contaminate food and water sources. The hazardous substances infect the body when come into the contact with the skin, eyes, or inhalation of the contaminated air. In this case, the territory on which, because of exposure to hazardous chemicals, massive damage to people, animals and plants occurred is called the focus of the chemical damage.

An important characteristic of the focus of the chemical damage formed by the potent poisonous substances (PPS) is the duration of the existence of the areas of the direct outflow (emission) of the substances, i.e. the persistence of the contamination. Most PPS with a boiling point of up to $20{ }^{\circ} \mathrm{C}$ (chlorine), as a rule, evaporate quickly, so the persistence of contamination in the areas of their outflow (emission) is not great.

However, vapours of such substances, including those in hazardous concentrations, can be detected at large distances (up to several kilometres) from the place of their outflow (emission). The wind has a great influence on the evaporation of the PPS, therefore, in settlements, the persistence of the contamination with them will be higher than in the open areas. In addition, in settlements and forests, stagnation of the air contaminated with the PPS vapours is possible, the formation of relatively high concentrations of vapours in basements, communication tunnels [4]. Chlorine is a greenish-yellow gas with a pungent odour; it is 2.5 times heavier than air. A cloud of chlorine moves in the direction of the wind, pressing to the ground, accumulating in lowlands, basements, tunnels. In open areas, the damaging concentrations of chlorine during the destruction of unborded containers of $50-100$ tons can spread during inversion at a distance of about $80 \mathrm{~km}$, with convection at a distance of $2-3 \mathrm{~km}$. The dumping containers, using degassing substances - neutralizers, which can reduce or prevent the intensity of evaporation, can significantly reduce the dimensions of hazardous areas during the spread of hazardous chemicals. If the outflow (emission) of the hazardous chemicals is accompanied by fire, then in such a situation the main task is to limit the spread of fire and at the same time take actions to rescue people from burning buildings [1].

\subsection{The area of the research practical application}

To take the adequate and timely measures to eliminate the accidents with the release of chlorine, the most accurate results of calculations of the main indicators are necessary, such as the depth of the zone of contamination of the potent poisonous substances, the amount of the poisonous substance in the primary and secondary clouds, the area of the contamination, etc. It is generally known [5], the method the calculation of these indicators is based on the use of the look-up tables and the coefficient method, which are convenient enough for the manual method. However, in the emergencies, when the serious threats to the life and health of people arise, and the situation can deteriorate rapidly, there is an acute shortage of time for a general assessment of the situation, making decisions on the organization of the rescue measures. It is obvious that the staff simply will not have enough time for the scrupulous calculations, and a stressful situation, in addition, with a high degree of probability will provoke serious errors and inaccuracies.

It seems the most expedient to develop an automated system capable of simulating scenarios of the potential contamination in the event of accidents with the release of the PPS. The preliminary research and forecasting of the possible scenarios will allow carrying 
out the preparatory measures, and in the event of the emergency, much faster and objectively assessing the main quantitative characteristics of the release of the PPS.

The software was developed in the Borland Delphi 7. The interface of the program that simulates scenarios of the potential contamination in case of the disruption of the technological process of the water chlorination in the urban environment is shown in Figure 1. As input values, the user needs to:

- select the storage conditions for the PPS (gas, compressed gas or liquid), in tons;

- choose the degree of the vertical stability of the air (inversion, isothermy or convection);

- indicate the wind speed in $\mathrm{m} / \mathrm{s}$ and the air temperature in Celsius degree;

- the density of the PPS in $\mathrm{t} / \mathrm{m} 3$ is set by the default for chlorine to 1.553 , but the user can change the value to the density of the another hazardous substance;

- indicate the thickness of the PPS in m;

- enter the distance from the source of contamination in $\mathrm{km}$;

- enter the time from the moment of the accident, min.

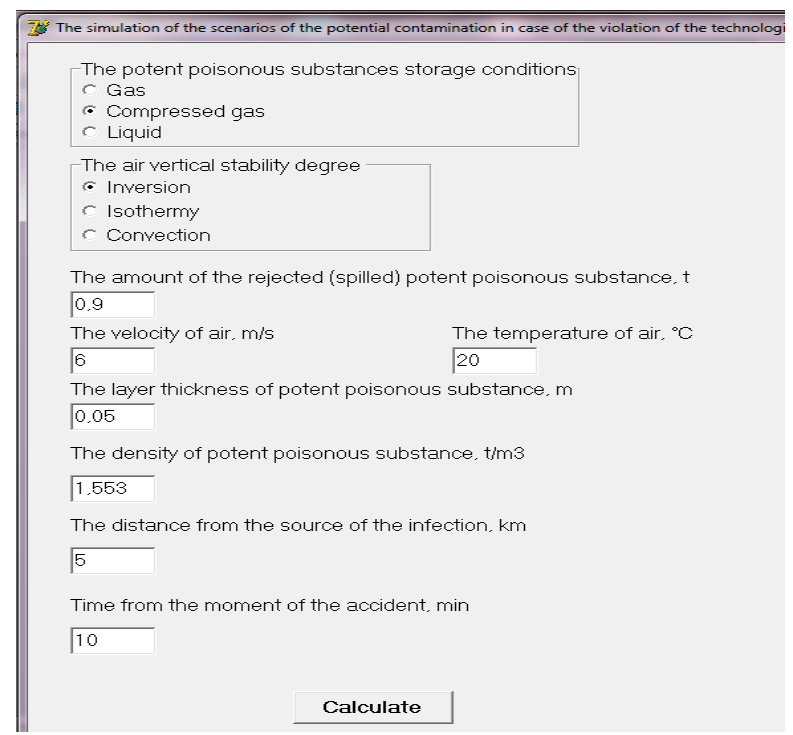

Fig. 1.The input of the initial data

Figure 2 showsthe calculation results. The quantitative values are assessed taking into account the characteristics described below.

1. The equivalent amount of the substance in the primary and secondary clouds (in tons), estimated according to the formulas:

$$
\begin{gathered}
Q e_{1}=K_{1} \cdot K_{3} \cdot K_{5} \cdot K_{7} \cdot Q_{0} \\
Q e_{2}=\left(1-K_{l}\right) \cdot K_{2} \cdot K_{3} \cdot K_{4} \cdot K_{5} \cdot K_{6} \cdot K_{7} \cdot\left(Q_{0} / h \cdot d\right)
\end{gathered}
$$

$Q_{э_{1}}$ - the equal quantity of the substance in the primary cloud, tones;

$Q_{\ni 2}$ - the equal quantity of the substance in the secondary cloud, tones;

$K_{l}$ - the coefficient depending on the storage conditions of the PPS;

$K_{2}$ - the coefficient depending on the physicochemical properties of the PPS;

$K_{3}$ - the coefficient equal to the ratio of the threshold toxic dose of chlorine to the threshold toxic dose of the another PPS;

$K_{4}$ - the coefficient taking into account the wind speed; 
$K_{5}$ - the coefficient that takes into account the degree of the vertical stability of air (it is taken equal for the inversion -1 , for the isotherm -0.23 , for the convection - 0.08 );

$K_{6}$ - the coefficient depending on the time elapsed after the start of the accident $\mathrm{N}$ (the value is determined after calculating the duration of the evaporation of the substance T);

$K_{7}-$ the coefficient taking into account the effect of the air temperature;

$Q_{0}$ - the amount of the PPS ejected (spilled) during the accident, ton;

$h$ - the layer thickness of the PPS, m;

$d$ - the density of the PPS, $\mathrm{t} / \mathrm{m}^{3}$.

2. The depth of the infestation by the primary and secondary clouds. The calculation of the depths of the zones of the contamination by the primary (secondary) cloud of the PPS in case of the accidents at the technological tanks, storage facilities and transport is carried out using special tables [6]. At the same time, the reference tables, as a rule, are given for the integer or interval values, which requires the specialist performing calculations of the necessary skill in order to correctly select the reference values of the coefficients. The developed software provides a linear approximation of the reference coefficients for any input values, which makes it possible to exclude errors or professional incompetence of the user, as well as significantly reduce the calculation time.

3. The total depth of the contamination $(\mathrm{km}$, due to the action of the primary and secondary clouds of the PPS) is estimated by the formula:

$$
D=D_{\max }+0.5 D_{\min }
$$

$D$ - the total depth of the contamination;

$D_{\max }$ - the maximum depth of the contamination;

$D_{\min }$ - the minimum depth of the contamination.

This value is compared with the maximum possible value of the depth of the transfer of the air masses $D_{m . p .}$. determined by the formula:

$$
D_{m . p .}=N \cdot V
$$

$N$-the time from the beginning of the accident, hours;

$V$ - the speed of the transfer of the leading edge of the contaminated air at the given wind speed and the degree of the vertical stability of the air, $\mathrm{km} / \mathrm{h}$.

4. The areas of the zones of the possible $\left(S_{p}\right)$ and actual $\left(S_{a}\right)$ contamination are estimated according to the formulas:

$$
\begin{aligned}
& S_{p}=8.72 \cdot 10^{-3} \cdot D^{2} \cdot \varphi \\
& S_{a}=K_{8} \cdot D^{2} \cdot N^{0.2}
\end{aligned}
$$

$D$ - the depth of the contaminated zone, $\mathrm{km}$;

$\varphi$ - the angular dimensions of the zone of the possible contamination, deg.;

$K_{8}-$ the coefficient, depending on the degree of the vertical stability of the air (assumed to be: the inversion - 0.081; the isothermy - 0.133; the convection - 0.295).

5. The time $(t)$ of the approach of the PPS cloud to the given object is estimated by the formula:

$$
t=x / V
$$

$x$-the distance from the source of the contamination to the given object, $\mathrm{km}$.

6. The duration of the damaging effect of the PPS $(T)$ is determined by the time of its evaporation from the spill area:

$$
T=(h \cdot d) /\left(K_{2} \cdot K_{4} \cdot K_{7}\right)
$$



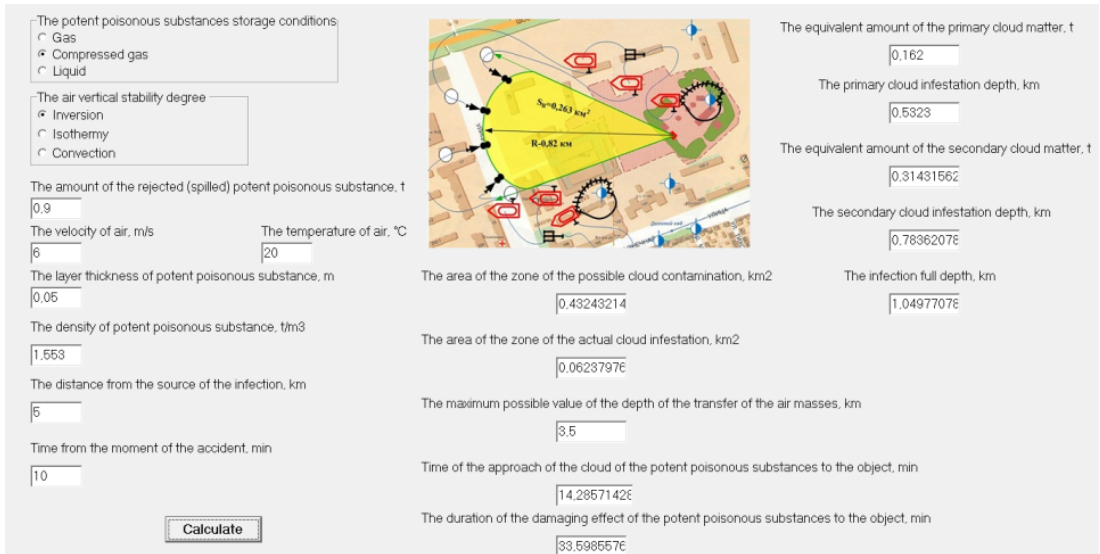

Fig. 2.The simulation results

The automation of the calculations makes it possible to conduct deeper studies of the various dependencies of the main indicators characterizing the emergency. In particular, the dependences of the duration of the damaging action on the wind speed and air temperature were established. It is shown in Figure 3.

Figure 4 shows a series of graphs of the dependences of the equivalent amount of matter and the depth of the affected area of the secondary cloud on the wind speed and the air temperature under the various conditions.

Figure 5 shows a series of graphs of the dependences of the area of the possible and actual PPS contamination on the air temperature under the various conditions.

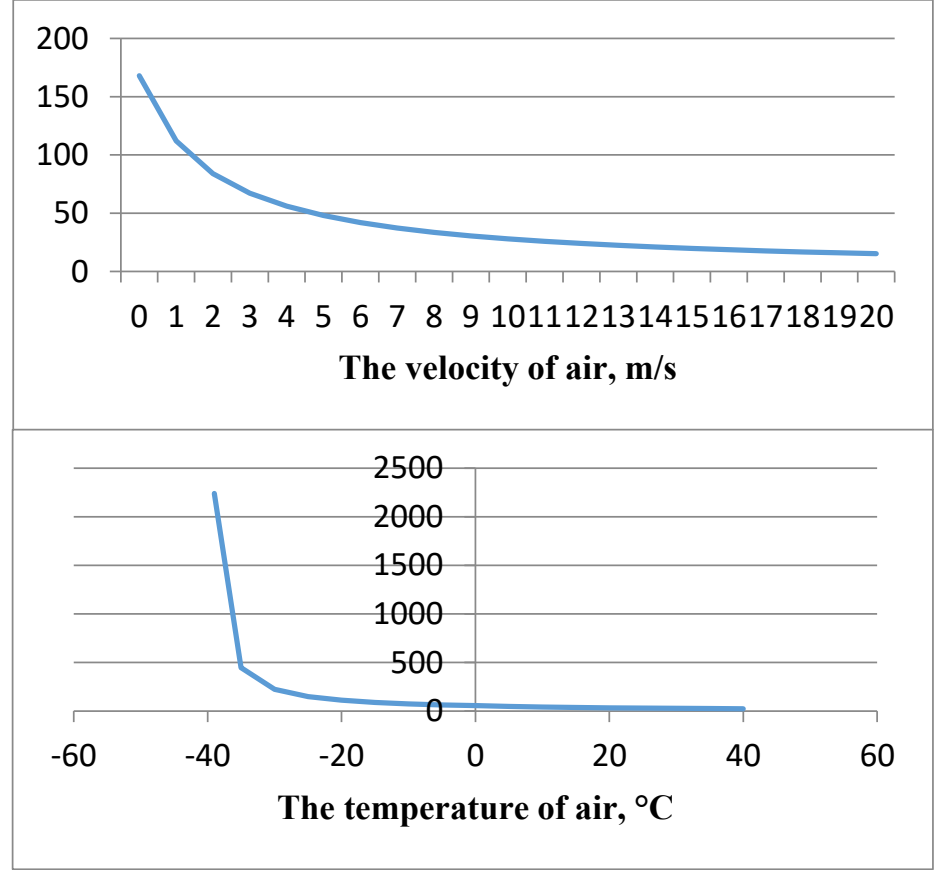

Fig. 3. The dependences of the duration of the damaging effect on the wind speed and the air temperature under the following parameters: the storage conditions of the PPS - gas, the amount of ejected substance $-0.9 \mathrm{t}$, the degree of the vertical stability of the air - inversion, the thickness of the PPS - $5 \mathrm{~cm}$, the time elapsed from the moment of the accident - 30-40 minutes, the distance from the source of the contamination $-5 \mathrm{~km}$ 


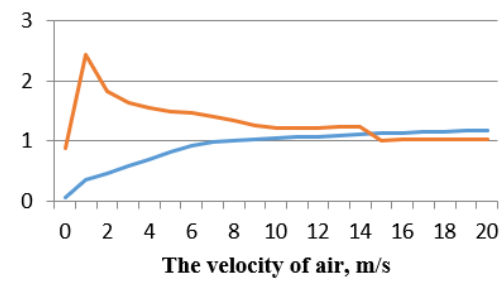

a

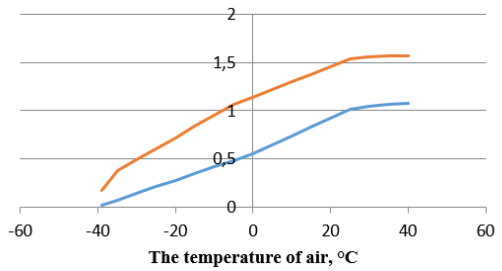

$\mathrm{c}$

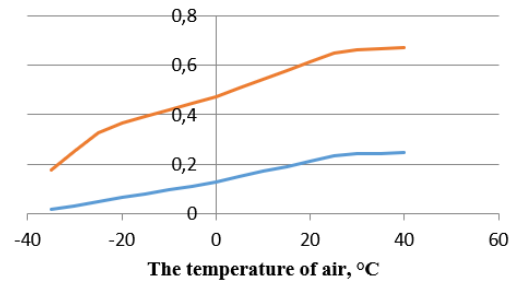

e

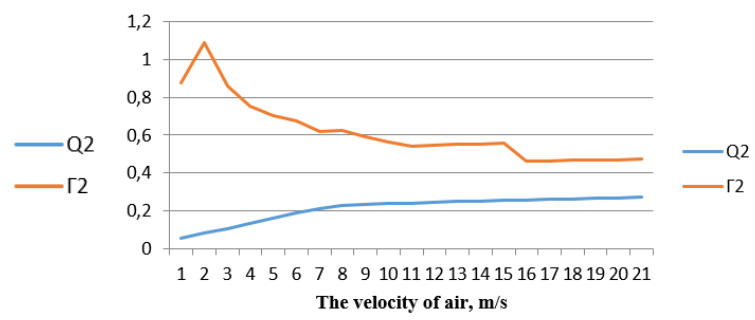

b

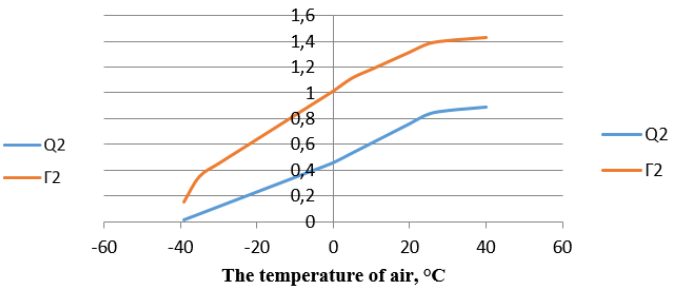

d

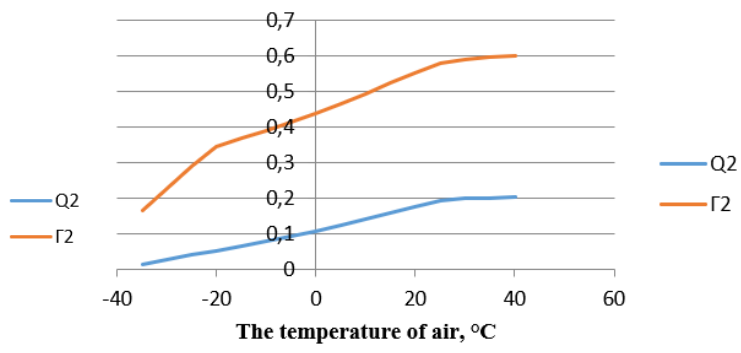

f

Fig. 4. The dependences of the equivalent amount of the substance $Q_{2}$ and the depth of the affected zone $D_{2}$ of the secondary cloud on the wind speed and the air temperature with the following parameters: the amount of the ejected substance $-0.9 \mathrm{t}$, the thickness of the PPS $-5 \mathrm{~cm}$, the distance from the source of the contamination - $5 \mathrm{~km}$,

a) the storage conditions of the PPS - gas, time elapsed from the moment of accident - 40 min, degree of the vertical stability of the air - inversion, temperature $-10^{\circ} \mathrm{C}$,

b) the storage conditions of the PPS - gas, time elapsed from the moment of the accident - $40 \mathrm{~min}$, degree of the vertical stability of the air - isotherm, temperature $-10^{\circ} \mathrm{C}$,

c) the storage conditions of the PPS - gas, time elapsed from the moment of accident - $30 \mathrm{~min}$, degree of the vertical stability of the air - inversion, wind speed $6 \mathrm{~m} / \mathrm{s}$,

d) the storage conditions of the PPS - compressed gas, time elapsed from the moment of the accident $30 \mathrm{~min}$, degree of the vertical stability of the air - inversion, wind speed $6 \mathrm{~m} / \mathrm{s}$,

e) the storage conditions of the PPS - gas, time elapsed from the moment of the accident - $30 \mathrm{~min}$, degree of the vertical stability of the air - isotherm, wind speed $6 \mathrm{~m} / \mathrm{s}$,

f) the storage conditions of the PPS - compressed gas, time elapsed from the moment of the accident $30 \mathrm{~min}$, degree of the vertical stability of the air - isotherm, wind speed $6 \mathrm{~m} / \mathrm{s}$ 


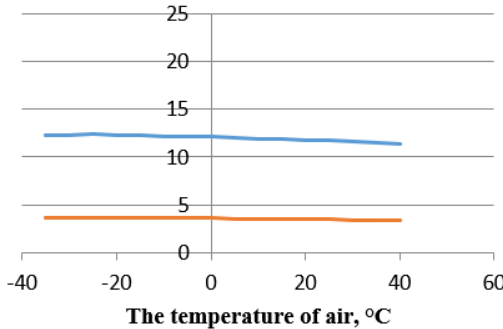

a

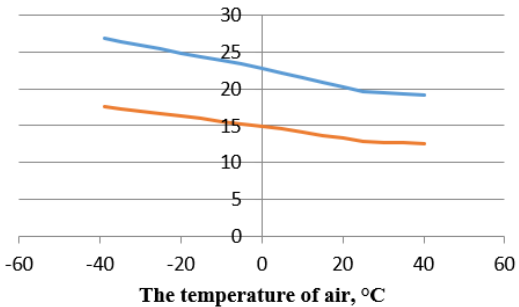

c

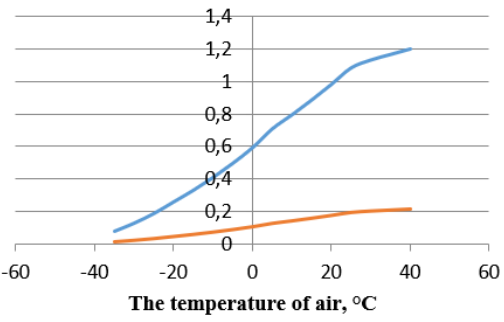

e

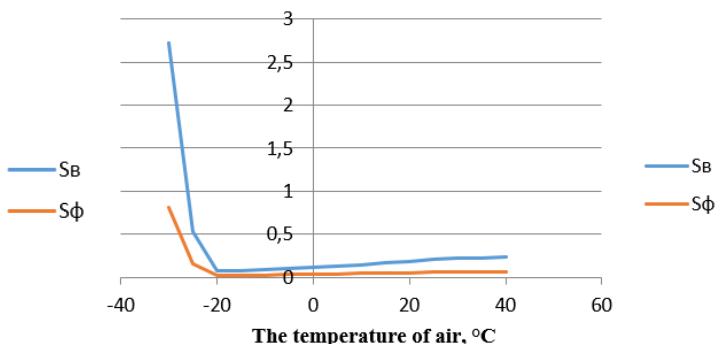

$\mathrm{b}$

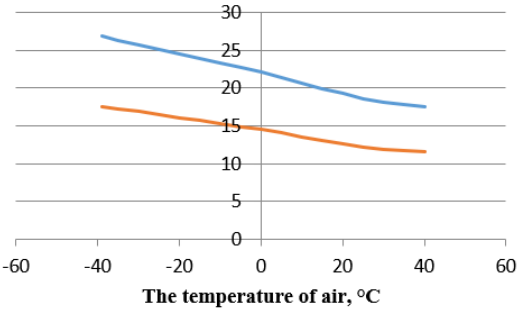

$\mathrm{d}$

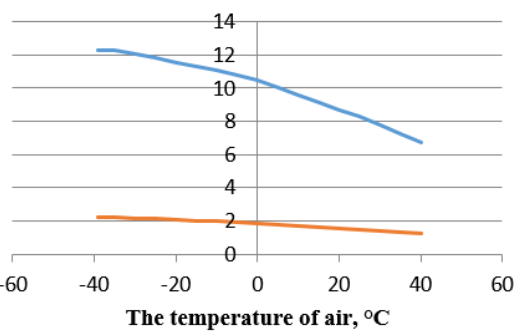

$\mathrm{f}$

Fig. 5. The dependences of the area of possible $S_{p}$ and actual Sa of the PPS contamination on the air temperature with the following parameters: the amount of ejected substance $-0.9 \mathrm{t}$, the PPS thickness $-5 \mathrm{~cm}$, distance from the source of the contamination $-5 \mathrm{~km}$, wind speed $-6 \mathrm{~m} / \mathrm{s}$, time elapsed since the accident $-30 \mathrm{~min}$.

a) the storage conditions of the PPS - gas, the degree of the vertical stability of the air - isothermal,

b) the storage conditions of the PPS - compressed gas, the degree of the vertical stability of the air isothermal,

c) the storage conditions of the PPS - gas, the degree of the vertical stability of the air - convection,

d) the storage conditions of the PPS - compressed gas, the degree of the vertical stability of the air convection,

e) the storage conditions of the PPS - compressed gas, the degree of the vertical stability of the air inversion,

f) the storage conditions of the PPS - gas, the degree of the vertical stability of the air - inversion

Therefore, the scale in the chemically hazardous accidents depends very much on the meteorological situation and the storage conditions of the PPS. Of all the degrees of the vertical stability of the atmosphere, inversion is the most favourable for eliminating the PPS cloud. The area of the emission of the PPS cloud during the isothermy will be 5 times less than during the inversion, and during the convection it will be 5 times less than during the isothermy. A high wind speed quickly dissipates the damaging concentrations of the PPS, and a lower speed reduces the depth of the contamination. The area of the emission of the PPS cloud in a closed region (in settlements with the compact standing buildings) will be 
approximately 3.5 times less than in an open area, with the appropriate degree of the vertical air stability and the wind speed. It is expedient to use the calculated parameters investigated by us when carrying out measures for the implementation of the protection of the civilian population outside the sanitary protection zones (SPZ) and caught in the emergency zone.

\section{Conclusions}

Accordingly, the developed automated system, which simulates the scenarios of the potential contamination in case of the disruption of the technological process of the water chlorination in the urban environment, makes it possible to calculate all the main quantitative characteristics of the release of the PPS. Furthermore the use of the automated system allows to get the result more quickly than with the traditional approach, eliminates errors caused by the human factor. The advantage of using the developed software is the ability to calculate approximating values from the look-up tables, so that the user can use the arbitrary (not necessarily integer) data for the input values. In addition, the study of the various dependencies (for example, the duration of the action of the source of the contamination or the depth of the contamination zone on the weather conditions and the storage conditions of the PPS) makes it possible to formulate more precise instructions for carrying out the rescue measures. The scheme of the experiment for simulation the various scenarios depending on the change in the input parameters included a comprehensive study, and in the article, due to the limited scope, only some of the most interesting for practical conclusions of dependence are presented.

\section{References}

1. V I Korolev,Methods of special training and tactical-special exercises with nonmilitary formations of civil defense ( Moscow, Voenizdat, 1986)

2. AN Denisov, MMDanilov, OI Stepanov, EEZaitsevaTactical methods of emergency reconnaissance and salvation when extinguishing fires(Moscow, Academy of State Fire Service of the Ministry of Emergency Situations of Russia, 2020)

3. SSTimofeeva, TI Drozdova, GV Plotnikova, VF GolchevskyPhysical and chemical foundations of development and extinguishing fires(Irkutsk, Publishing house of ISTU, 2013)

4. A M Kostrov, Civil defense(Moscow, Education, 1991)

5. S Yu Kapustin, V IMalakhov,Methodological guide for forecasting and assessing the chemical situation in emergency situations (Ivanovo, AtomIzdat, 2001)

6. V I Korolev,Everyone should know and be able to do this: Instruction for the population(Moscow, Voenizdat, 1980) 